\title{
LA ESCENOGRAFÍA EN EL TEATRO CABALLERESCO
}

\author{
María GutiÉRrez PADilla \\ Universidad Nacional Autónoma de México \\ mariagutierrezpadilla@gmail.com
}

$\mathrm{E}$ s sabido que, en general, algunas comedias que se representaban en palacio contaban con una escenografía más elaborada, en contraste con aquellas de los teatros comerciales, y esto por los elementos técnicos con los que se podía contar y la posibilidad de representar en exteriores, como en jardines o el estanque del Buen Retiro (Díez 2002: 46-71). Pero las puestas en escena espectaculares, en cuanto a elementos escenográficos, suelen estar vinculadas a un tipo determinado de comedia: «En las de capa y espada, la escenografía era mínima [...]. Por el contrario, en las comedias de santos, en las de magia y en las mitológicas, la escenografía era mucho más complicada» (Alonso 2007: 19); pero, ¿qué tantos decorados y tramoyas exigen las comedias caballerescas? Debido a que falta un estudio crítico que plantee algunos requerimientos escenográficos del teatro caballeresco, en las siguientes páginas haré un análisis de algunos elementos. Me ceñiré a La Gridonia o el cielo de Amor vengado de Félix de Arteaga (Fray Hortensio Paravicino) para establecer de qué manera pudo haber sido llevada a escena, y contrastaré con El castillo de Lindabridis de Calderón de la Barca, lo cual permitirá establecer tendencias sobre la espectacularidad de la escenografía en el teatro caballeresco; cimentaré la propuesta a partir de las didascalias que ofrece la obra y las posibilidades técnicas con las que se podía contar.

La elección de las obras está supeditada a los elementos en común que presentan: ambas están basadas en un hipotexto de libro de caballerías, fueron representadas en espacios interiores en palacio después de la llegada a España de los escenógrafos italianos y fueron puestas en escena durante el reinado de Felipe IV. La Gridonia o el cielo de Amor vengado y El castillo de Lindabridis pertenecen al corpus de textos que constituyen el teatro caballeresco, que Claudia Demattè define como «aquellas obras teatrales que reescriben la materia caballeresca

Edad de Oro, XXXIV (2015), pp. 131-143, ISSN: 0212-0429 - DOI http://dx.doi.org/10.15366/edadoro2015.34.008 
autóctona española quedando por tanto excluidas aquella carolingia o ariostesca, y centrándose en la reescritura de unos sesenta libros de caballerías, que constituyen el hipotexto» (2011: s. p.). Las dos obras dramatizan y adaptan episodios identificables de algún libro de caballerías: La Gridonia toma como hipotexto el Primaleón (1512), y El Castillo de Lindabridis el Espejo de príncipes y caballe$\operatorname{ros}(1555)^{1}$.

Existen pocos estudios críticos dedicados a La Gridonia, y una parte de los autores refiere su existencia sin analizar ningún aspecto (Cerdan 1979: 129; López 2007: 197). Algunos críticos han señalado las similitudes y diferencias que guarda la pieza dramática con el hipotexto (Calderón 2009: 21-22; Cerdan 2002: 156; Demattè 2005: 102-103). Manuel Calderón, en su introducción a la edición moderna de La Gridonia, señala algunos aspectos relevantes en cuanto a la contextualización de la puesta en escena, el tema, el estilo y la originalidad de los juegos escénicos que propone la obra (2009: 18-27). Francis Cerdan ofrece un análisis sobre el tipo de espacios que se dan en la pieza dramática, y encuentra que la mayoría de los cambios de espacio están indicados en el parlamento de los personajes (2002: 163).

En cuanto a El castillo de Lindabridis, existen algunos trabajos más, aunque no dejan de ser pocos en comparación con los dedicados a otras obras de Calderón de la Barca. Valbuena Briones comenta la relación de la obra de teatro con Espejo de príncipes y caballeros y propone una lectura alegórica a partir de dos elementos de la obra: la cueva del Fauno, que representa la fuerza de los sentidos, y el castillo volador, como la conciencia ascensional del alma en la contemplación de la belleza (1981: 373-383). En su investigación, Sandra Nielsen señala elementos orientales presentes en El castillo de Lindabridis, como los jardines colgantes y la elección de Babilonia para las escenas finales (1998: 92), pero no menciona el hipotexto, en contraposición con el trabajo de Ana Lorena Leija, quien centra parte de su investigación en señalar las similitudes entre El castillo de Lindabridis y Espejo de príncipes y caballeros, y posteriormente analiza la obra de Calderón desde un enfoque teatrológico (2009: 219-230). En otro estudio, Leija se centra en la denominación genérica que ha recibido El castillo de Lindabridis y propone que debe designarse como «teatro de espectáculo», debido a los elementos de espectacularidad: el fauno, la música, el baile y el desfile previo al torneo; el objetivo del trabajo es elaborar una propuesta de puesta en escena a partir de las nuevas tecnologías (2008: 93). Además, en un importante artículo, Susana Hernández Araico analiza la estructura de la obra y la función dramática que tienen algunos elementos; en cuanto a la escenografía, ella opina que se reduce a recursos elementales propios de las representaciones en palacio, aunque no los explica (2007: 67-77).

Para ahondar en las dinámicas de transtextualidad, véase Demattè 2011 y 2005: 37-45. 
Como se ha podido observar, los estudios sobre La Gridonia se enfocan en los tipos de espacio, los temas, el estilo y las semejanzas y diferencias que la obra guarda en relación con el hipotexto. En cuanto a la obra de Calderón, las tendencias críticas se orientan a distintos aspectos como lecturas alegóricas, posibles influencias para la creación de la pieza teatral, similitudes entre el hipotexto y la obra dramática; y solo se ha mencionado tangencialmente el aspecto escenográfico de El castillo de Lindabridis, por lo que en las siguientes páginas me centraré en las posibilidades escenográficas de la puesta en escena de La Gridonia en palacio y contrastaré los elementos con la pieza de Calderón; para lo cual, sigo a Alfredo Hermenegildo en sus consideraciones teóricas, con la distinción entre didascalias explícitas e implícitas; las primeras se refieren a aquellas

en que el autor enuncia, de manera directa y con indicadores precisos, ciertos gestos, mímica, movimientos, desplazamientos, apariciones, desapariciones o acciones de los personajes, o ciertas manipulaciones de objetos, que, unos y otras, deben formar un sistema de signos integrados de una manera obligatoria en la enunciación del discurso dramático. (2001: 56)

Las implícitas están insertas en el discurso dramático y son indicadas por medio de «ciertos gestos, mímica, movimientos, desplazamientos, apariciones, desapariciones o acciones de los personajes» (2001: 56).

En cuanto al corpus, empleo el impreso de La Gridonia que se incluye en Obras póstumas, divinas, y humanas de 1641; para la obra calderoniana, utilizo la edición que se conserva en la Biblioteca Nacional de España, disponible en la Biblioteca Cervantes Virtual. He seleccionado trabajar con estas ediciones debido a la consideración de José María Ruano de la Haza: «es fundamental que para la reconstrucción de la puesta en escena de una comedia el investigador utilice únicamente textos impresos o manuscritos del siglo XVII» (1988: 83), ya que con el acceso al texto aurisecular podemos tener una visión que no está parcializada por los editores modernos.

\section{LA GRIDONIA}

Es muy posible que La Gridonia haya sido representada en alguno de los salones de palacio después de 1623 (Calderón 2009: 22), por lo que probablemente el tablado haya sido construido específicamente para el evento o para una serie de representaciones, ya que fue escrita para celebrar visitas reales o algún cumpleaños (Calderón 2009: 18).

El escenario empleado para la representación de esta obra debió ser una construcción tablado-vestuario, en cuyo nivel inferior se hallaban puertas para la 
entrada y salida de los actores, y en la parte superior, los balcones de apariencias. Algo similar a la descripción del corral de Córdoba que hace Ángel María García Gómez: «En su pared frontera al patio había varias aperturas, posiblemente tres, que daban acceso a un balcón que corría por encima de las tres puertas que mediaban entre el vestuario bajo y el tablado» (1991: 187).

Es seguro que el escenario contara con las puertas del vestuario debido a las didascalias explícitas que lo confirman: «Entran y cruzan por unas puertas y otras huyendo y turbados, los cazadores»; «habrá salido por una puerta y vuelto a entrar por otra Rosicler» (f. 122v). A partir de esta disposición espacial debemos imaginar la distribución de los elementos escenográficos.

\subsection{El monte y los personajes mitológicos}

El monte fue un elemento escenográfico muy frecuente en numerosas comedias que se representaban en los corrales ${ }^{2}$, y no es de extrañar que también se recreara en una obra palaciega, sobre todo porque no presenta mayores dificultades de construcción: se trataba de una rampa con escalones o una rampa inclinada que baja del primer corredor, sobre las puertas, al tablado (Ruano 1994: 419-420, 426), por lo que los actores pueden subir y colocarse en el primer corredor, como lo debieron de hacer Artemidoro y Rosicler: «Artemidoro habrá subido a la parte prevenida del monte, donde se descubra el retrato de Gridonia con el león a los pies» (f. 134v); y ya en el corredor pudo haber descubierto manualmente la apariencia del retrato de Gridonia. En esta misma escena, Rosicler sube al monte mientras recita su parlamento para ver de cerca la imagen de la amada, y la didascalia explícita indica: «Artemidoro y el retrato desaparecen con mucha tramoya. Y Rosicler bajará, como despeñado, del lugar donde había subido» (f. 135r). Debido a que el actor desciende de manera abrupta, es posible que este monte se haya construido con una rampa.

En cuanto a la desaparición del retrato, pudo haberse dejado caer el lienzo pintado para crear el efecto. La desaparición de Artemidoro, «con mucha tramoya», es muy distinta: lo más probable es que se haya empleado el bofetón, «una especie de torno que giraba 180 grados, sobre un eje vertical a gran velocidad, de forma que era capaz de hacer aparecer o desaparecer rápidamente a un actor o a cualquier objeto» (Alonso 2007: 26). El bofetón estaría colocado en el primer corredor, en la cima del monte.

Esta misma tramoya se emplea en dos ocasiones más. Después de que Rosicler baja del monte, queda solo en el tablado y escucha una voz con la que dialoga; el actor debió haber caminado hasta el otro extremo del escenario, donde «descubre

\footnotetext{
Para profundizar en las comedias en las que se empleó el monte como elemento escenográfico, véase Ruano 1994: 474-484.
} 
Dafnes en la forma que la pintan comúnmente convirtiéndose en aquel árbol [laurel]» (f. 136v). Considero que se empleó el bofetón porque después del parlamento de Dafne se lee la didascalia explícita: «Cúbrese la tramoya». El bofetón debió girar, porque Rosicler quedó solo en escena nuevamente, y «vase turbado a asegurar de una peña, ábrese, y muéstrase Anaxarte convertida (con toda imitación del vestido) en mármol» (f. 137r); es el mismo caso que Dafne. La aparición y desaparición abrupta de los personajes que solo se muestran para vaticinar el incumplimiento del amor contribuye a la espectacularidad de la puesta en escena, y la tramoya más indicada para crear este efecto es el bofetón, que también se empleaba en los teatros comerciales (Alonso 2007: 26).

\subsection{Los vuelos de los personajes}

A lo largo de la obra hay tres vuelos de personajes. En la segunda escena: «Baja una Ave Fénix como la describe Claudiano, un rayo en el pico y en las uñas, a Artemidoro en traje de pastor galante»; «Deja el Fénix en tierra a Artemidoro y vuelve a esconderse al cielo» (f. 123r). Estos movimientos verticales de escenografía eran muy empleados tanto en el teatro comercial $^{3}$ como en el de palacio, en especial en obras mitológicas y comedias de santos para el vuelo de los personajes; de esta manera, las tramoyas se convirtieron en elementos «indispensables para producir los signos escénicos necesarios, que originan una variada gama de significados con el denominador común de lo mítico, maravilloso, sobrenatural» (Díez 2002: 96). La máquina que permite este tipo de juegos escénicos es el pescante o canal, que «consistía en un madero vertical en el cual se había practicado una hendidura o canal que, convenientemente engrasada, permitía que una peana horizontal se deslizase hacia arriba o hacia abajo» (Alonso 2007: 27); pero debido a que en la representación no vuelve a emplearse, pues el resto de los vuelos se indican en trayectoria diagonal, pienso que en esta escena pudo haberse utilizado una maquinaria más sofisticada: «una especie de grúa articulada y giratoria compuesta por un soporte vertical o pie derecho y una pieza horizontal, denominada deslizador [...] donde cabalgaban los personajes» (Rodríguez 1989: 56). El movimiento se lograba por medio de un juego de ruedas, engranajes y poleas que pendían desde el techo del escenario. Así, en esta escena el deslizador tendría un decorado del ave para dar el efecto de que el actor va montado en ella.

Más adelante, «aparecerá Artemidoro en lo alto dél [teatro], en el traje que escogiere, irá dando vuelta al monte» (f. 145v). Dado que la didascalia no expresa que el actor esté sobre algún elemento escenográfico, es posible que se haya empleado una tramoya similar al garabato, aprovechando la instalación del primer vuelo: «dos ganchos de fierro que se fijaban a dos presillas reforzadas cosidas en

3 Para ahondar en las comedias en las que se empleó el pescante, véase Ruano 1994: 474-484. 
las hombreras del traje del actor» (Alonso 2007: 28). El vuelo se efectuaría por medio de una serie de poleas, garruchas y cuerdas suspendidas sobre el escenario, esta tramoya permite hacer vuelos en cualquier dirección (Ruano 1998: 160): así Artemidoro rodearía el monte. El garabato también era empleado en los teatros comerciales (Alonso 2007: 28), por lo que esta escena habría podido recrearse en un corral de comedias.

Posteriormente, se encuentran Artemidoro, Florisbella, Sirene y Primaleón, y Artemidoro les indica: «Retiraos los tres solos a la tienda» (f. 181v). Esa tienda debió de estar simulada en la puerta del centro del vestidor, pues los personajes en escenas anteriores entran y salen por ahí: «Entra Sirene a la tienda» (f. 153r); en una didascalia implícita, Sirene ordena «Recógete a la tienda» (f. 166v), y la didascalia explícita anuncia: «vase Primaleón» (f. 167r); «Primaleón y Sirene salen de la tienda» (f. 172v). En la escena que refiero, Paravicino indica que Artemidoro se eleva (f. 182r); mientras Artemidoro vuela con la primera tramoya descrita en este apartado, el parlamento de Armelinda dará la idea al espectador del vuelo de la tienda: «una tienda de campaña, / dentro del parque ha plantado» (f. 183r). De haberse presentado en un teatro comercial, este vuelo habría tenido que efectuarse mediante la canal.

\section{EL CASTILLO DE LINDABRIDIS}

Ya para la década de $1660^{4}$, el teatro cortesano había experimentado una evolución ${ }^{5}$. Generalmente se representaba en el Coliseo del Buen Retiro o, como El castillo de Lindabridis, en los salones o saloncetes del palacio; la gran diferencia con las representaciones de 1600 radica en el cambio de concepción del espacio escénico, diseñado de acuerdo al modelo italiano: cuenta con un proscenio, y bastidores colocados en perspectiva por donde los representantes salen a escena, por lo que permite el uso de decorados que crean la ilusión de profundidad y en general el empleo de maquinaria que posibilita mayor espectacularidad escénica (González 2002: 132). Esto no implica que todas las comedias concebidas para este espacio tuvieran las mismas características escenográficas; de las cerca de ochenta piezas representadas en los palacios y jardines reales entre 1621 y 1665 , el 90\% de ellas no eran de tramoya, «son o parecen ser comedias de las que se escenificaban o podían escenificarse en los corrales» (Pedraza 1998: 76). Este debió ser el caso de El castillo de Lindabridis, una comedia escrita específicamente para palacio, pero adaptable a los corrales.

\footnotetext{
El castillo de Lindabridis se representó hacia 1661 (Valbuena 1981: 376).

Para ahondar en el tema, véase Ruano 1998: 161.
} 
En esta obra calderoniana, los elementos escenográficos más espectaculares se encuentran en las primeras dos jornadas; en la tercera se desarrolla el enredo de confusión de identidades debido a los disfraces entre Lindabridis y Claridiana, por lo que la escenografía pierde relevancia ante la intriga que se desarrolla. Para el análisis, me centraré únicamente en algunos elementos escenográficos como el castillo y el jardín interior, porque considero que son los que mejor ejemplifican la posibilidad de adaptación al corral.

\subsection{La escenografía general}

El fondo del escenario debía tener un decorado fijo con una escenografía rocosa, y en el tablado tuvo que haber una cueva, porque, como considera Ruano de la Haza:

Podemos estar seguros de que se utilizaron decorados para la representación de una comedia, solamente si 1) son mencionados y descritos explícitamente en las acotaciones; o 2) se alude a ellos en el diálogo y poseen una importancia temática en la obra o son utilizados de manera práctica por los actores. (1988: 86)

La cueva es referida por los personajes y es el sitio donde el Fauno se quedará encerrado luego del aterrizaje del castillo.

La obra inicia con tres caballeros en escena, después se revelará que son Rosicler, Floriseo y un criado, e inmediatamente después aparece el Fauno, como la didascalia explícita señala, «vestido de pieles y con un bastón grande y nudoso, lo más extraño y feroz que pueda» $(1)^{6}$; la vestimenta del fauno lo identifica con un ser salvaje, reforzado por el accesorio escénico del bastón: como comenta Abel Alonso Mateos, «una de las funciones más características de los accesorios escénicos es la de identificar desde distintos ángulos al personaje que los utiliza: procedencia, status social, profesión u oficio» (2007: 38). Así, tanto la escenografía como la configuración física del Fauno crean un ambiente montañoso o rocoso, que, además, estaría reforzado por las didascalias implícitas «esta lóbrega montaña», en boca del Fauno, y «peligro d'estos montes», dicho por Rosicler.

La cueva era un elemento escenográfico muy usual en los corrales, sobre todo para comedias de santos, por lo que no resulta extraño que estuviera presente en palacio. La cueva, al igual que las rocas, es «un decorado convencional para el que se utilizaban ramas y plantas con la adición de rocas — seguramente de cartón pintado armado sobre un bastidor» (Ruano 1994: 409). Sin embargo, también cabe la posibilidad de que la cueva fuera una apariencia pintada en uno de los

6 Dado que el impreso disponible no cuenta con una numeración de los versos, pagino el PDF desde la portada con el número 1 y así sucesivamente; por lo que para citar, en lo sucesivo, lo haré por páginas. 
bastidores del fondo del escenario. Lo que es un hecho es que la cueva se encontraba presente en el escenario, como lo confirma la didascalia implícita por medio del deíctico demostrativo: «te espero en esta cueva obscura» (2). Después el Fauno debió de salir del escenario por la entrecalle o claro que se forma entre los bastidores, justo delante de la cueva, para dar la idea de que el castillo lo dejará encerrado, como se verá más adelante ${ }^{7}$.

\subsection{El castillo y el jardín}

Sin dudas, el elemento escenográfico más espectacular de la obra es el castillo volador de Lindabridis. La primera didascalia explícita que refiere a este elemento indica «descúbrese el castillo» (3); dado que después aparecen otras dos didascalias, «baja el castillo» y «asiéntase el castillo, y abren la puerta» (4), es de suponer que lo que se descubre es la parte baja del castillo, suspendida por una tramoya.

En el escenario en perspectiva, el tipo de tramoya para vuelos verticales que pudo haberse empleado "consistía en un cilindro enejado en un árbol o madero redondo, alrededor del cual se enroscaba una maroma. Uno de los extremos de esta maroma se ataba a un torno y del otro se suspendía un peso o plomada. En el mismo árbol se enroscaba una segunda maroma, de la cual colgaba la tramoya» (Ruano 1998: 159). Este mecanismo se afianzaba por encima del escenario, a diferencia de lo que ocurría en los teatros comerciales, donde, como se vio anteriormente, se empleaba la canal o pescante. En el escenario de corral, la adaptación no debería ser demasiado compleja, aunque la canal habría de estar montada desde antes de que se empleara; podría ocultarse tras una cortina, como se hizo en la representación de El condenado por desconfiado (Ruano 1994: 474).

Antes de que el castillo baje, se crean expectativas en el espectador gracias a las didascalias implícitas reveladas en voz de Floriseo: «el gran prodigio que en el aire veo» y después «aterriza el castillo» (4). La espectacularidad podía ser reforzada por elementos sonoros al momento del aterrizaje. Cuando el castillo ya se encuentra asentado en el escenario, Floriseo menciona: «posado [el castillo] en la roca / que a la cueva del fauno abrió la boca, / le deja sepultado, / seguro el monte ya, y a ti [Rosicler] vengado» (4); como se ha visto, el fauno salió de escena, pero basta el parlamento del personaje para transmitir la idea del encierro.

La espectacularidad escenográfica del castillo no solo radica en el vuelo vertical, sino que tiene puerta, ventana y jardín interior. Calderón indica: «asiéntase el castillo, y abren la puerta»; y otra didascalia dicta: «salen todas las damas que puedan, Sirene, Arminda y Lindabridis, vestidas ricamente» (4). Una vez posado

Existen otras obras cortesanas en las que aparecen decorados de cuevas, como El premio de la hermosura de Lope de Vega. Aunque su escenificación no fue en un espacio cerrado, se revela que era un elemento escenográfico muy recurrente tanto en corral como en palacio. 
el castillo en el escenario, las damas debieron de haber salido detrás de él, por el foro; las entradas de los actores a escena generalmente se hacían entre los bastidores, pero en casos excepcionales también podían salir por el foro, como ocurre en Fieras afemina amor de Calderón (Ruano 1998: 156). Así, el castillo debía tener en la parte inferior una abertura que semejara una puerta para las salidas de las damas, como lo confirma otra didascalia explícita casi al final de la segunda jornada: «ábrese el castillo, como primero, y salen las damas» (36); esta puerta o cortinilla permite que los comediantes aparezcan en escena creando la ilusión de que salen de la construcción, lo que aumenta la espectacularidad de la representación. La puerta también se emplea en otra escena: el castillo acaba de llegar a Constantinopla y se abre la puerta para que Claridiana salga, y sostiene un diálogo con Lindabridis, quien después «éntrase y cierra la puerta» (31), según reza la didascalia explícita correspondiente.

En la posibilidad de una representación en corral, este movimiento sería muy complicado, pero podía adaptarse: las damas saldrían al tablado por el vestuario, junto al castillo, y se crearía la idea de que salen del edificio según la didascalia implícita indicada por Rosicler: «un pasmo a otro sucede, pues, abiertas / del castillo veloz las altas puertas, / un escuadrón de ninfas se me ofrece» (4). Tras la salida de las damas, hay un breve diálogo en escena y después otra didascalia explícita: «vase Lindabridis» (7). La princesa se iría del tablado por el mismo sitio por el que entró, simulando que regresaba a su morada, ya que una de sus doncellas, Sirene, detiene a Rosicler, quien quiere seguirla: «Detente; / estos umbrales no pises» (7).

Entonces, «salen a la ventana del castillo Lindabridis, Sirene y Arminda» (8) para ver combatir a los caballeros que están en el tablado; las doncellas dialogan con ellos, lo que implica que el castillo debió de tener un espacio que simulaba la ventana, y que las damas se encontraban detrás de este elemento escénico. Resultaría espectacular que la ventana se encontrara sobre la puerta y que las doncellas dialogaran a una altura superior con los caballeros, pero no he encontrado documentación sobre cómo se lograría este efecto; sin embargo, pudieron haber subido por una escalerilla detrás del castillo, o incluso la ventana podía estar ubicada junto a la puerta y la escena haberse desarrollado a la altura del tablado. En el caso del corral, las damas estarían colocadas detrás del castillo en uno de los corredores de la fachada (Ruano 1994: 373).

En la segunda jornada, se lee una didascalia explícita que indica: «ábrese el castillo, y salen como a un jardín que estará fingido: dentro dél Lindabridis y las damas, dejando abierta la cueva del fauno» (18). Esto se lograría con el cambio del telón de foro: «con dos bastidores grandes, y sin recortar, cuyos raíles se unían en el centro del tablado visible; es decir, en el lugar del punto de fuga de la perspectiva» (Ruano 1998: 157); el mismo recurso emplearía Calderón en Fieras 
afemina amor: se trata de un bastidor que se abre para mostrar otro decorado, lo que implicaría que entre jornadas se elevaba el castillo y se simulaba por medio de un decorado, que en la escena se abriría para mostrar el jardín. Es posible que no se recrease con accesorios, como sugiere la indicación de Calderón: «fingido»; entonces únicamente estaría pintado. En cuanto a la posibilidad del corral, entre jornadas también se levantaría el castillo, y el jardín se mostraría en el espacio central del nivel inferior del fondo del escenario, descorriendo una cortina (Ruano 1998: 409).

Esta escena se compone del espacio interior del jardín, donde están las damas (al fondo del escenario), y el exterior, donde se ubican los coros que cantan a Lindabridis (cerca del proscenio, un coro a la derecha y otro a la izquierda). La princesa se queda dormida en el jardín y las doncellas salen de escena para escuchar mejor la música; entonces «sale el fauno por la cueva» (20), este personaje seguramente salió al tablado por la entrecalle inmediata donde está pintada la boca de cueva del foro. El juego espacial se ve reforzado por la didascalia implícita puesta en el parlamento de Lindabridis: «Cómo te atreviste, cómo, / a profanar la clausura / de un castillo, donde el sol, / si entra, entra con la disculpa / de que viene a traer el día» (20). Lindabridis, ofendida por la transgresión del fauno, llama a los caballeros para que la defiendan, ellos salen a escena, pero permanecen fuera del castillo.

Después, Lindabridis «entra, y cierra el castillo, que desaparece, y queda el teatro como antes estaba» (23). Lo que esta didascalia indica es el cambio del telón de foro nuevamente, y el escenario queda con el primer decorado rocoso. Es en la tercera jornada cuando el castillo aparece en escena de nuevo; gracias a la didascalia «baja el castillo» (31), se puede suponer que se vuelve a emplear la tramoya para el descenso del elemento escenográfico.

\section{TENDENCIAS ESCENOGRÁFICAS DEL TEATRO CABALLERESCO}

En la obra de Paravicino se privilegian las didascalias explícitas sobre las implícitas, al contrario de lo que ocurre en la obra de Calderón, quien prefiere poner las indicaciones en voz de los personajes.

Considero que la espectacularidad en las obras de tipo caballeresco dependerá de los elementos que el dramaturgo haya seleccionado del hipotexto. En $\mathrm{La}$ Gridonia, Paravicino rescata la situación en que la protagonista quiere la cabeza de Primaleón y él se enamora de la doncella, por lo que abundan los parlamentos largos que reflejan los sentimientos de los personajes, quienes externan frecuentemente sus puntos de vista sobre temas relacionados con el amor y el matrimonio. En consecuencia, la acción se reduce a que algunos buscan a Primaleón para 
retarlo en combate o a que otros buscan a Gridonia para pedirla en matrimonio a cambio de la cabeza de Primaleón. Por otra parte, El castillo de Lindabridis centra gran parte de su acción en el enredo del triángulo amoroso entre Lindabridis, Claridiana y Febo.

Pedraza afirma que «son numerosas las comedias escritas específicamente para palacio, pero que no difieren sustancialmente de las escenificadas en los corrales. Con frecuencia presentaban un lenguaje y unas situaciones más elaboradas» (1998: 76). Considero que en este tipo de obras pueden englobarse las comedias analizadas, porque, como se ha podido observar, La Gridonia debió de haber empleado recursos escenográficos sofisticados disponibles en las representaciones de palacio, pero fácilmente adaptables a los teatros comerciales: el monte, el bofetón, la canal y el garabato. Es el mismo caso de El castillo de Lindabridis, una de las obras que, por sus características escenográficas, pudieron tener cabida en teatros comerciales: como se ha visto, los recursos escenográficos no exigen el uso de maquinaria exclusiva del palacio.

En la obra de Paravicino la espectacularidad escenográfica de la obra está cimentada en los vuelos de los actores y en la aparición y desaparición abrupta de los comediantes; en la de Calderón, en el castillo con su jardín interior. Pero es necesario considerar que también están presentes, en ambas comedias, otros componentes que complementan una espectacularidad visual, característica de las representaciones en palacio: los efectos sonoros, los decorados, el rico vestuario, por ejemplo.

Recibido: 11/09/2015

Aceptado: 13/10/2015

\section{BIBLIOGRAFÍA CITADA}

Alonso Mateos, Abel (2007). «El teatro barroco por dentro: espacios, escenografía y otros recursos de la comedia áurea». Per Abbat: Boletín Filológico de Actualización Académica y Didáctica, 2, pp. 7-46.

Arteaga, Félix de (1641). La Gridonia o el cielo de Amor vengado. En Obras póstumas, divinas y humanas, Madrid: Carlos Sánchez, 1641, ff. 121r-189r.

Calderón de la Barca, Pedro (1691). El castillo de Lindabridis. [En línea]. Centro Virtual Cervantes. $<\underline{\text { http://www.cervantesvirtual.com/obra/el-castillo-de-lindabridis--0/> }}$ [Consulta: 24-06-2015]

CAlderón, Manuel (2009). «Estudio introductorio». En Félix de Arteaga (Fray Hortensio Paravicino), La Gridonia, Madrid: Consejo Superior de Investigaciones Científicas, 2009. 
Cerdan, Francis (1979). «Bibliografía de fray Hortensio Paravicino». Criticón, 8, pp. 1-149. (2002). «Espacio natural y espacio prodigioso en La Gridonia de fray Hortensio Paravicino». En François Cazal (ed.), Homenaje a Frédéric Serralta. El espacio y sus representaciones en el teatro español del Siglo de Oro. Madrid: Universidad de Navarra-Iberoamericana-Vervuert, pp.155-168.

Demattè, Claudia (2011). «Teatro caballeresco y segundones: crónica de una pasión no solo literaria por los libros de caballerías». [En línea]. Centro Virtual Cervantes. $<\mathrm{http} / /$ www.cervantesvirtual.com/obra/teatro-caballeresco-y-segundones-cronica-de-unapasion-no-solo-literaria-por-los-libros-de-caballerias/> [Consulta: 24-06-2015] (2005). Repertorio bibliografico e studio interpretativo del teatro caballeresco spagnolo del sec. XVII. Trento: Dipartamento di Scienze Filologiche e Storiche.

DíEz BorQue, José María (2002). Los espectáculos del teatro y de la fiesta en el Siglo de Oro. Madrid: Laberinto.

García Gómez, Ángel María (1991). «La casa de las comedias de Córdoba», Cuadernos de Teatro Clásico, 6, pp. 177-196.

González, Aurelio (2002). «Los espacios del Barroco en Calderón». En Aurelio González (ed.), Calderón 1600-2000. Jornadas de investigación calderoniana. México: El Colegio de México, Centro de Estudios Lingüísticos y Literarios: Fondo Eulalio Ferrer, pp. 59-77.

Hermenegildo, Alfredo (2001). Teatro de palabras. Didascalias en la escena española del siglo xvi. Lleida: Edicions de la Universitat de Lleida.

HernáNDEZ Araico, Susana (2007). «Las inverosimilitudes imaginativas de Calderón y su función dramática teatral: El castillo de Lindabridis». Teatro de Palabras: Revista sobre Teatro Áureo, 1, pp. 67-77.

LeIJA, Ana Lorena (2008). «Enredos y fantasía: los géneros en la comedia El castillo de Lindabridis de Calderón de la Barca». Anuario de Literatura Dramática y Teatro, 1, pp. 87-95.

(2009). «La guerra civil y el torneo a muerte en El castillo de Lindabridis de Calderón de la Barca: adaptación y realización escénica». Anuario Calderoniano, 2, pp. 219230.

LóPez MARTínez, María Isabel (2007). «Cernuda, Paravicino y el Greco: menage á trois», Anuario de Estudios Filológicos, 30, pp. 195-211.

NIELSEN, Sandra (1998). «Lindabridis y su castillo volante: el medievalismo y el orientalismo en una comedia novelesca de Calderón». En Jules Whicker (coord.), Actas del XII Congreso de la Asociación Internacional de Hispanistas (21-26 de agosto de 1995), vol. 3. Birmingham: University of Birmingham, Department of Hispanic Studies, pp. 90-93.

Pedraza JimÉneZ, Felipe (1998). «El teatro cortesano en el reinado de Felipe IV». En José María Díez Borque (dir.), Teatro cortesano en la España de los Austrias, Madrid: Compañía Nacional de Teatro Clásico, pp. 75-104.

RodríGUEZ G. DE CEBALlos, Alfonso (1989). «Escenografía y tramoya en el teatro español de siglo xvii». En Aurora Egido (coord.), La escenografía del teatro barroco, Salamanca: Universidad de Salamanca-Universidad Internacional Menéndez Pelayo, pp. 33-60. 
(1988). «Hacia una metodología para la reconstrucción de la puesta en escena de la comedia en los teatros comerciales del siglo xvii». Criticón, 42, pp. 81-102.

Ruano de la Haza, José María (1998). «La escenografía del teatro cortesano». En José María

Díez Borque (dir.), Teatro cortesano en la España de los Austrias. Madrid: Compañía Nacional de Teatro Clásico, pp. 137-168.

(1994). Los teatros comerciales del siglo xvii y la escenificación de la comedia. Madrid: Castalia.

VALbuena Briones, Ángel (1981). «La influencia de un libro de caballerías en El castillo de Lindabridis». Revista Canadiense de Estudios Hispánicos, 5, 3, pp. 373-383.

La escenografía en el teatro caballeresco

Resumen: En el presente artículo, estudio algunos requerimientos escenográficos del teatro caballeresco, para establecer tendencias sobre la espectacularidad de la escenografía en este tipo de obras. Los resultados serán presentados a partir del análisis de las didascalias en La Gridonia o el cielo de Amor vengado de Fray Hortensio Paravicino y El castillo de Lindabridis de Calderón de la Barca.

Palabras clave: teatro palaciego, escenografía, materia caballeresca, Calderón, Paravicino.

\section{Scenography in the chivalry theater}

ABSTRACT: In this paper, I study some scenographic requirements of the theater of chivalry themes, to establish trends about the spectacular scenery in this kind of plays. Results are presented from the analysis of: La Gridonia o el cielo de Amor vengado by Fray Hortensio Paravicino and El castillo de Lindabridis by Calderón de la Barca.

KeYwords: Palace Theater, scenography, chivalric theme, Calderón, Paravicino. 
TERESA DE JESÚS (1515-1582)

Guillermo Serés (Universidad Autónoma de Barcelona)

La imaginación de Santa Teresa: virtudes y desatinos de «la loca de la casa»....... 11

Ana Garriga EsPino (Universidad Autónoma de Madrid)

El desafío editorial de las cartas de Teresa de Jesús.

LeCTURAS REPUBLICANAS DEL QUIJOTE

RAQUEL ArIas (Universidad Autónoma de Madrid)

León Felipe alista a don Quijote en el bando republicano. 57

MANUEl LóPez ForJas (Universidad Autónoma de Madrid)

El Quijote y la realidad viva de las Españas: la visión histórica

de Pedro Bosch-Gimpera.

CArmen Madorrán Ayerra (Universidad Autónoma de Madrid)

El Quijote como utopía necesaria: la mirada de Adolfo Sánchez Vázquez 85

\section{OTROS TEMAS}

SònIA BoADAS (Universitat Autònoma de Barcelona)

Libros y librerías: la recepción de Commynes en España 101

Lucila Lobato Osorio (Universidad Nacional Autónoma de México)

La noticia de la muerte de Policisne de Boecia: expresiones de dolor en un libro inconcluso.

María Gutiérrez Padilla (Universidad Nacional Autónoma de México)

La escenografía en el teatro caballeresco 131

ADRIÁn FERnÁNDEZ (Université de Fribourg)

Dos entremeses «auditivos» y sus niveles: hacia un metateatro visual. 145

David González de la Higuera Garrido (Universidad Complutense de Madrid) La visión premonitoria de Alonso en El caballero de Olmedo:

otra recuperación de lo popular

Esther Fernández LóPEz (UNED)

Perseo en la comedia tardobarroca: Ignacio Ferrera y Pasqual 169

Manuel Piqueras Flores (Universidad Autónoma de Madrid)

De La hija de Celestina $a$ La ingeniosa Elena: estructura narrativa, género literario e interpolación 
Dagoberto CÁceres Agullar (Western University Canada)

Realismo incesante: desde Celestina hacia su recepción 201

TeXTOS INÉDITOS

Davinia Rodríguez Ortega (Universidad Pública de Navarra)

Auto sacramental del Juego del Hombre, de Luis Mejía de la Cerda:

edición crítica 


\section{EDAD DE ORO}

Revista de Filología Hispánica XXXIV

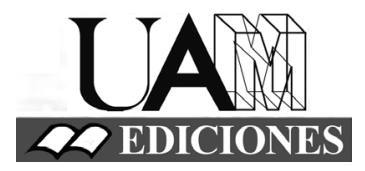




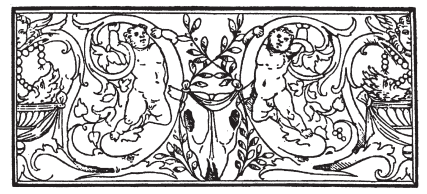

\section{Edad de Oro. Revista de Filología Hispánica}

ISSN: 0212-0429

Dirección:

Teodosio Fernández

Secretaría y edición:

José Ramón Trujillo

Comité científico internacional:

Carlos Alvar (Univ. de Ginebra)

Ignacio Arellano (Univ. de Navarra)

Javier Blasco (Univ. de Valladolid)

Alberto Blecua (UAB)

Jean Canavaggio (Univ. de París X)

Laura Dolfi (Univ. de Turín)

Aurora Egido (Univ. de Zaragoza)

Víctor García de la Concha (RAE)

Luciano García Lorenzo (CSIC)

Joaquín González Cuenca (Univ. de Castilla-

La Mancha)

Agustín de La Granja (Univ. de Granada)

Begoña López Bueno (Univ. de Sevilla)

Michel Moner (Univ. de Toulouse III)

Joan Oleza (Univ. de Valencia)

Alfonso Rey (Univ. de Santiago)

Lina Rodríguez Cacho (Univ. de Salamanca)

Leonardo Romero Tobar (Univ. de Zaragoza)

Aldo Ruffinatto (Univ. de Turín)

Lía Schwartz (City University of New York)
Redacción y admisión de originales:

Teodosio Fernández

Edad de Oro

Departamento de Filología Española

Universidad Autónoma de Madrid

28049 Madrid (España)

Tfno.: +0034914974090

correo: teodosio.fernandez@uam.es

Distribución, suscripción y venta:

Servicio de Publicaciones de la UAM

Universidad Autónoma de Madrid

28049 Madrid (España)

Intercambio de publicaciones:

Biblioteca de la Facultad de Filosofía y

Letras (UAM)

Universidad Autónoma de Madrid

28049 Madrid (España)

Han colaborado en este volumen:

Departamento de Filología Española (UAM)

Facultad de Filosofia y Letras (UAM)

Edad de Oro se recoge, entre otras, en las siguientes bases de datos: SCOPUS, MLA Database, HLAS, Latindex, PIO-Periodical Content Index, ISOC, Dialnet, MIAR, ERIH, DICE, Sumaris CBUC, Ulrich's. Se encuentra evaluada en CIRC: A; INRECH; MIAR difusión ICDS live: 9.977; SCImago Journal \& Country Rank: H Index 2, SJR 0,101, Q4; RESH índice de impacto: 0.041; ERIH: A INT1; Carhus Plus+ 2014: C. 